Bruce Seaman, "¿Qué está en juego al optar entre distintas formas de apoyo para el sector cultural?" Estudios Públicos 146 (2017).

\title{
EL PATRIMONIO DE LA CIUDAD ENTENDIDO COMO UN VALOR. CÓMO SE REGULA Y SE INCENTIVA ESE VALOR
}

\author{
Elke Schlack \\ Pontificia Universidad Católica de Chile
}

\begin{abstract}
RESUMEN: Retomando lo que Seaman plantea sobre la incidencia de la protección patrimonial en la valoración económica de barrios o de una ciudad, este comentario describe la experiencia que tenemos en Chile con normativas que han condicionado aumentos de valor. Los incentivos a la iniciativa privada en la protección patrimonial y la calidad urbana también se comentan, con el fin de derivar preguntas sobre hacia quién y con qué fin están concebidos estos instrumentos.

PALABRAS CLAVE: protección patrimonial, espacio público, barrios, incentivos, financiamiento privado.
\end{abstract}

\section{URBAN HERITAGE CONSIDERED AS VALUE. HOW THIS VALUE IS REGULATED AND INCENTIVIZED}

ABSTRACT: Taking up Seaman's proposition about the positive effects that safeguarding heritage can have on the economic value of urban districts or a city, this commentary describes Chile's experience with regulations that have created the conditions for increases in value. Incentives for private enterprise to safeguard heritage and the quality of the urban fabric are also touched on with a view to raising

Elke Schlack. Arquitecta por la Pontificia Universidad Católica de Chile y doctora en arquitectura por la Universidad Tecnológica de Berlín. Email: eschlack@uc.cl.

* Versión revisada del comentario hecho a la conferencia de Bruce Seaman expuesta el 24 de noviembre de 2016 en el CEP y publicada en este número de Estudios Públicos. 
some questions about who these instruments are aimed at and to what purpose.

KEYwords: heritage protection, public space, urban districts, incentives, private financing.

B ruce Seaman nos convoca a una muy interesante discusión sobre lo que está en juego cuando decidimos entre distintas formas de apoyar la preservación del patrimonio a través de normativas urbanas y otros instrumentos, como subsidios, impuestos e incentivos. Su presentación apunta a un tema crucial en el desarrollo urbano. ¿Cómo se financia la protección patrimonial y quiénes son sus destinatarios? Un tema que habitualmente se discute en el ámbito de la protección de la cultura o el patrimonio edificatorio, acá es abordado por Seaman de una manera mucho más holística desde las políticas urbanas.

La cultura modifica los valores de la ciudad. En el ámbito urbano, con esto estamos diciendo que el valor cultural de un barrio le otorga un valor económico agregado a su valor habitual como edificio o suelo urbano. Este fenómeno se ha ido haciendo más patente en el desarrollo urbano desde los años noventa, momento en que se ha perfilado un cambio en el que la localización de las grandes y pequeñas inversiones ya no se basa sólo en aspectos físico-espaciales o funcionales de la ciudad, sino también en el atractivo de barrios y ciudades en el contexto de una competencia mundial (Fainstein 2001; Florida 2003; Zukin 1995). En ese ámbito, el valor patrimonial edificatorio de los barrios y ciudades juega un rol importante, pero también la cultura en forma de eventos, ferias, expos y actividades de turismo, como ha ocurrido con ciudades como Barcelona gracias a las olimpiadas, en Lisboa con la Expo, en París y Londres con su oferta turística tradicional y en Nueva York o Berlín con su connotación vinculada a la industria creativa y el turismo cultural. Bruce Seaman relaciona, entonces, la discusión sobre el financiamiento de la cultura y el patrimonio urbano no sólo con patrimonios edificatorios, sino que también vincula su argumentación a fenómenos como el turismo urbano y explica que, más allá del fenómeno de la ciudad creativa — que atrae la economía de los jóvenes empresarios de la industria creativa-, hay otras cualidades de los barrios, como sus espacios públicos y aspectos, que aportan a su calidad de vida y pueden implicar un aumento en la valorización de un lugar en la ciudad. 
Uno de los desafíos que se presentan en la regulación de bienes patrimoniales es el logro de un equilibrio de oportunidades para los diferentes beneficiados. Ése es el tema que plantea Bruce Seaman cuando nos muestra a modo de ejemplo el desequilibrio que se produce en la valorización del patrimonio a través de declaraciones de la Unesco. Explica que instituciones de este tipo al definir el valor patrimonial de un barrio implícitamente inciden en la valorización de éste. Como hay un número mayor de declaraciones patrimoniales de la Unesco en ciudades de Europa y en el norte del mundo, según Seaman se estarían generando desigualdades, castigando a ciudades del sur del mundo, con menos declaratorias.

Una valorización del patrimonio puede desencadenarse debido a diversos instrumentos. A través de normativas internacionales, tal como lo está planteando Seaman, y también por normativas a nivel local, las cuales pueden tener una importante incidencia en los valores de suelo o de los inmuebles. Esto puede ocurrir debido a declaraciones de "zona típica" o de "conservación histórica" que se definen para barrios determinados, o también por definiciones de uso y tipo de edificación en los planes reguladores. En Chile aún faltan estudios empíricos suficientes como para formarnos un juicio claro sobre el impacto que tiene la declaración de una zona típica o de conservación histórica sobre los precios de un barrio. Por un lado, las declaratorias pueden congelar el valor de los inmuebles debido a las fuertes restricciones de restauración a las que están sujetos, haciéndolos poco apetecibles para sus residentes, ya que deben pagar altos costos de mantención. Por otro lado, en zonas patrimoniales con potencial comercial, podría haber un aumento de demanda por patrimonio, cuando el valor patrimonial de un inmueble puede ser capitalizado para usos determinados vinculados con el turismo, por ejemplo.

Este último aspecto lo hemos estudiado de manera más exhaustiva en el caso del barrio Italia-Caupolicán en Santiago de Chile. Se trata de un barrio con valor patrimonial. Sin estar formalmente declarado como zona típica o de conservación histórica, la normativa comunal impone un gravamen indirecto de protección patrimonial, a través de la limitación de la altura de edificación. En el contexto del desarrollo inmobiliario de Santiago, que se caracteriza por una normativa altamente permisiva, la limitación de altura en el barrio Italia hace poco probable que se 
desarrollen proyectos que impliquen la demolición de edificios en el barrio. Al mismo tiempo, la normativa es muy permisiva en cuanto a tipos de usos de suelo, lo cual abre la posibilidad de destinar el lugar a usos no residenciales. Como es de esperarse, según argumentaciones como la de Ingram (2006), el lugar comenzaría a cambiar de destino residencial a un destino comercial. El valor del uso comercial del barrio es lo suficientemente alto como para justificar que no es necesario demoler para sacarle más provecho económico a ese suelo.

Un valor adicional simbólico otorgado a los terrenos ubicados en el barrio Italia se genera a partir de la declaración del Consejo Nacional de la Cultura y las Artes, que define el barrio Italia como el "Barrio del Diseño". Esta iniciativa pública no hace sino apoyar algo que espontáneamente se había estado desarrollando en el lugar: las antiguas casas comienzan a ser capitalizadas por emprendimientos de gastronomía y tiendas de diseño, conformándose en un atractivo de turismo urbano nacional e internacional.

Se pudo comprobar que los valores de suelo en el sector central del barrio Italia aumentaron entre el año 1990 y el 2013 de 0,14 a 0,23 UF por metro cuadrado, mientras que en la comuna de Providencia se mantuvieron estables en un promedio de $0,45 \mathrm{UF} / \mathrm{m}^{2}$ (Schlack y Turnbull 2015).

Considerando que habitualmente todos los espacios centrales de las ciudades están sometidos a la presión de un cambio de uso (Ingram 2006), al menos en el desarrollo de este barrio la normativa y la declaración de "Barrio del Diseño" contribuyeron a que la valorización del lugar fuera más acelerada. También queda claro que gran parte del desarrollo y valorización de este sector depende de decisiones espontáneas y libres de diversos emprendimientos individuales que se fueron sumando. Un rol importante lo tuvieron el temprano desarrollo en el recinto industrial llamado Factoría Italia y el posicionamiento de diversos emprendimientos de arte, coleccionismo y antigüedades existentes en el lugar desde los años ochenta.

Otro de los desafíos que se plantean para la protección patrimonial, según Seaman, es la forma de incentivar el desarrollo de condiciones que contribuyan a la calidad urbana. Entre otros ejemplos, él se refiere a los espacios públicos y cómo ellos redundan sobre la atracción que ese tipo de barrios pueden generar para determinados grupos de 
habitantes. Muchas veces la provisión de espacios públicos es iniciativa de las políticas públicas, sin embargo, Seaman se refiere aquí a la posibilidad de que privados se involucren más en la protección o provisión de espacios públicos o paisajes que aporten a la calidad de vida mediante incentivos.

La experiencia que podemos aportar al respecto dice relación con un estudio sobre la regulación de espacios públicos provistos por privados en la comuna de Providencia. Se trata de una cláusula del plan regulador de esa comuna, por medio de la cual se ofrece un incentivo a aquellos proyectos inmobiliarios que destinen superficies del primer piso para el uso público. El premio consiste en mayores derechos de constructibilidad, lo cual puede repercutir en un aumento de hasta 200 por ciento de metros cuadrados construibles dependiendo del caso. La rentabilidad adicional que esta normativa otorga a los proyectos inmobiliarios ha implicado que se construyeran, entre 1976 y 2008, 62 proyectos con cesiones de tránsito público en el primer piso. En total, suman casi 60 mil metros cuadrados de superficie, que se ocupan tanto como patios semipúblicos de edificios de oficinas o como galerías comerciales de alta intensidad peatonal, que casi se confunden con el espacio público de las veredas de la comuna.

En el estudio realizado de estos 62 casos se analizó la concurrencia peatonal de los espacios y mediante entrevistas a los gestores y sus arquitectos fue posible concluir que el incentivo en sí no necesariamente garantizaba la ejecución de espacios que fueran útiles al uso público. Casi la mitad de los gestores involucrados en los proyectos estaban convencidos del valor que pasajes y patios interiores les aportarían a sus proyectos inmobiliarios, e incluso algunos aumentaban la cantidad de cesiones exigidas como mínimo para consolidar mayores superficies lineales de vitrinas. Sin embargo, la otra mitad no veía sentido en dicha política de espacio público, salvo por la mera posibilidad de obtener una ganancia adicional de metros construibles. En esos casos, los espacios resultantes eran áreas de baja calidad, a menudo pasajillos entre medianeros ciegos, que no revestían ningún interés al paisaje y calidad peatonal del lugar. Finalmente, varios de estos pasajes malogrados fueron cerrados.

Con este estudio quedó comprobado que el incentivo funciona bien en la medida en que se defina el objetivo con un alto grado de precisión. 
En este caso, el objetivo era el logro de un espacio público peatonal públicamente accesible y destinado al uso también público. En tanto el objetivo no estaba claramente formulado por el plan regulador o por los seccionales, ocurría que el gestor del proyecto condicionaba la calidad del espacio como una resultante de otras decisiones que le parecían más importantes, como, por ejemplo, una mejor distribución de los recintos interiores o un diseño que fuese fácil de mantener cerrado al tránsito público.

Las normativas cumplen un rol muy relevante en condicionar la protección de patrimonio o bienes públicos. Aunque son instrumentos destinados a regular aspectos técnicos, como altura o uso de suelo, definen también el valor de un barrio y pueden contribuir a que este valor se proteja. Estas normativas también pueden producir desigualdades en la asignación de valores a un barrio y no a otro (Fainstein 2001; Price 2016). Y pueden desencadenar procesos de expulsión de unos residentes por otros, o de un tipo de economías locales por otras. Es por eso que es necesario aclarar para quién y para qué es el beneficio de la protección del patrimonio urbano en dichas normativas. ¿Quiénes son los que se benefician de las definiciones normativas? ¿Son los dueños del suelo, los arrendatarios o los que compran un suelo para realizar un proyecto? Una vez que se haya entendido cómo cada uno de esos actores se ve afectado o beneficiado por la protección patrimonial será posible lograr estrategias realmente sustentables, con las cuales las políticas de protección patrimonial contribuyan a una equidad urbana.

\section{REFERENCIAS}

Consejo Nacional de la Cultura y las Artes (CNCA). 2012. "Barrio Italia - Barrio del Diseño". Santiago: Consejo Nacional de la Cultura y las Artes.

Fainstein, S. 2001. "Competitiveness, Cohesion and Governance: Their Implications for Social Justice". International Journal of Urban and Regional Research 25 (4): 884-88.

Florida, R. 2003. The Rise of the Creative Class. New York: Basic Books.

Ingram, G. 2006. "Patrones del desarrollo metropolitano ¿qué hemos aprendido?" En Santiago: dónde estamos, hacia dónde vamos, editado por Alex Galetovic. Santiago: Centro de Estudios Públicos.

Price, J. J. 2016. "Racionalidad económica de la política cultural”. Estudios Públicos 144 (2016): 165-197. 
Schlack, E., ed. 2015. POPS- El uso público del espacio urbano. El carácter público y la normativa. Santiago: Ediciones ARQ UNAB Capital Books.

Schlack, E. \& N. Turnbull. 2015. "Emerging Retail Gentrifications in Santiago de Chile: The Case of Italia-Caupolicán”. En Global Gentrifications, editado por L. Lees, H. Shin \& E. López-Morales. Bristol: Policy Press University of Bristol.

Seaman, B. 2017. “¿Qué está en juego al optar entre distintas formas de apoyo para el sector cultural?”. Estudios Públicos 146.

Zukin, S. 1995. The Cultures of Cities. Cambridge, MA: Blackwell. EP 